\title{
Dosimetric comparison of lateral opposing fields in moderate-to-severe active TAO retro-orbital irradiation
}

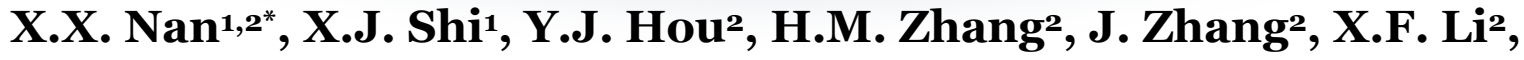 \\ Y.Y. Gao ${ }^{*}$ \\ ${ }^{1}$ Department of Radiotherapy, Beijing Luhe Hospital, affiliated to Capital Medical University, Beijing, China \\ ${ }^{2}$ Department of Radiotherapy, First Hospital of Shanxi Medical University, Shanxi, China
}

\begin{abstract}
- Original article

Background: To evaluate and compare the dosimetric advantages of lateral opposing fields (LOF) and intensity-modulated radiation therapy (IMRT) in moderate-to-severe active thyroid-associated ophthalmopathy (TAO) retroorbital irradiation. Materials and Methods: Fifty-eight TAO patients who underwent radiotherapy from 2012 to 2018 were chosen. LOF and IMRT plans were separately developed for each patient. The independent samples $t$-test was used to compare the differences among conformity index $(\mathrm{Cl})$, homogeneity index (HI), dose received by the maximal 95\% of the target (D95), and organs-at-risk (OAR) doses between the two groups. Pearson's correlation analysis was used to analyze the relationship between exophthalmos and OAR dose. Results: $\mathrm{Cl}$ and $\mathrm{HI}$ in the IMRT group were better than those in the LOF group, but crystal, eyeball, and optic nerve dose were higher than those in the LOF group $(P<0.05)$. There was no significant difference in $\mathrm{D} 95(\mathrm{P}=0.261)$. In the LOF group, exophthalmos was negatively correlated with crystal and eyeball dose $(P=0.000)$. However, there was no correlation between these indicators in the IMRT group $(P>0.05)$. In the LOF group, the median observation time was 26 months; the CT value of post-ball adipose tissue, exophthalmos, and clinical activity score was lower than those before treatment $(P=0.000)$, and eyesight was better than that before treatment $(P=0.000) ; 10 \%(2 / 20)$ had dry eyes and $5 \%(1 / 20)$ had decreased vision. Conclusions: LOF is safer than IMRT and can make full use of the high exophthalmos of TAO patients to reduce OAR dose.
\end{abstract}

Keywords: Tyroid associated ophthalmopathy, graves ophthalmopathy, retro-orbital irradiation, lateral opposing fields, safety evaluation.

\section{INTRODUCTION}

Thyroid-associated ophthalmopathy (TAO), also known as Graves ophthalmopathy, is an autoimmune disease that mainly affects the orbit. And its incidence rate accounting for the first among all orbital diseases (1). The European Group on Graves' Orbitopathy has provided detailed grading standards and treatment recommendations for the disease (2). High-dose glucocorticoids are still first-line therapy for patients with moderate-to-severe TAO (3); however, several clinical studies have shown that retrobulbar radiotherapy is a safe and effective treatment option, especially for patients in whom glucocorticoid therapy is ineffective (4-8). Nonetheless, the use of this therapy is still controversial, because TAO is a benign disease, and the patients' lenses have very low tolerance to radiation, which has raised concerns about the side effects of radiotherapy (9).

Although the successive appearance of three-dimensional conformal radiotherapy 
(3DCRT) and conventional intensity-modulated radiotherapy (IMRT) has allowed clinicians to precisely adjust the dose distribution (10-14), due to the particularity of the anatomic structure behind the ball, precision radiotherapy techniques such as IMRT may not be as advantageous as expected. However, lateral opposing fields (LOF) may play a stronger protective role to take advantage of the exophthalmos of TAO patients can draw the lens away from the target area to better protect endangered organs. Clinical research based on the characteristics of TAO has remained blank. For this reason, this study compared the dose distribution and adverse reactions from these two irradiation techniques for treating TAO retrobulbar radiotherapy during moderate and severe active periods.

\section{MATERIALS AND METHODS}

\section{Case data}

Fifty-eight patients with moderate-to-severe active TAO, who were treated with radiotherapy at the First Hospital of Shanxi Medical University (Shanxi, China) from April 2012 to May 2018, were enrolled in this study. The study population comprised 33 males and 25 females, aged 32-72 (median age, 42) years. All patients had previously failed first-line hormone therapy (orbital emission computed tomography (ECT) $(+)$, clinical activity score [CAS] score $\geq 4$ points). All the procedures were approved by the ethics committee of Shanxi Medical University.

\section{Positioning, target area delineation, and plan formulation}

The patient's head was fixed with thermoplastic head film, and the scanning range was $5 \mathrm{~cm}$ above and below the orbital area, obtaining the computed tomography (CT) images that were transmitted to the Eclipse Treatment Planning System version 8.9 (Varian Medical Systems, Palo Alto, CA) for exophthalmos measurement (figure 1). This was followed by delineation of the target area and formulation of the plans. The clinical target volume (CTV) encompassed the fat space behind the ball and all extraocular muscles. The anterior border was the outer iliac crest and the eyeball. The upper, lower, and lateral sides were the orbital wall, and the medial side was the ethmoid sinus wall. The CTV expanded forward and outward by $0 \mathrm{~mm}$, and the posterior, left, right, upper, and lower expanded by $3 \mathrm{~mm}$ to generate the planning target volume (PTV). The bilateral eyeballs, crystals, lacrimal glands, and optic nerves were delineated the endangered organs.

A 7-field IMRT and isocenter two-field lateral opposing field (LOF) radiotherapy plans were formulated, and wedge plates were used to adjust the dose. A lead block was used at the front of the target area to reduce the scattered rays, achieving a target area prescription dose $95 \%$ of the PTV (24 Gy/2Gy/12f; figure 1).

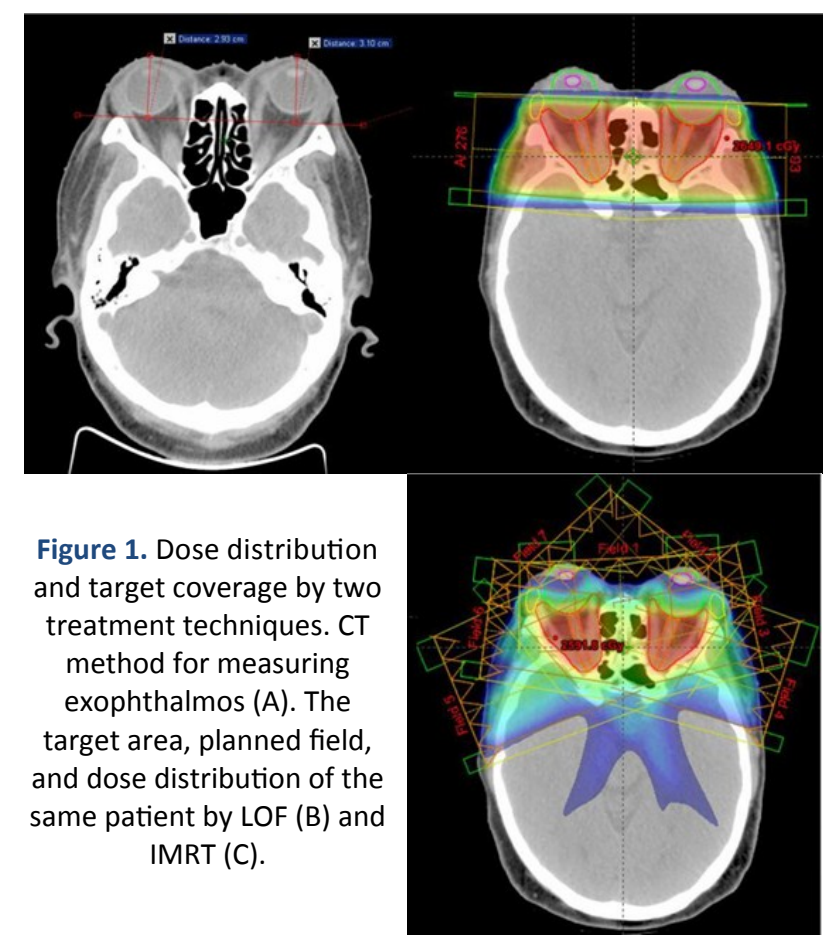

\section{Doseology evaluation indicators}

The CI and $\mathrm{HI}$ of the target area were compared, where $\mathrm{CI}=\mathrm{V}_{\mathrm{PTV}} \times \mathrm{T}_{\mathrm{TV}} / \mathrm{TV}_{\mathrm{PV}} \quad\left(\mathrm{V}_{\mathrm{PTV}}\right.$ : volume of PTV; $\mathrm{V}_{\text {TV }}$ : treatment volume included in the prescription isodose; $\mathrm{TV}_{\mathrm{PV}}$ : volume of the PTV in the prescription isodose) and HI = D5 / D95 (D5 and D95: maximum doses received at $5 \%$ and $95 \%$ volume of PTV). The following PTV dose parameters were calculated: Dmin, Dmax,

Int. J. Radiat. Res., Vol. 19 No. 2, April 2021 
Dmean, Dmedian, D95, D05, and D01, followed by evaluation of Dmin, Dmax, Dmean, Dmedian, D05, and D01 of endangered organs including bilateral lens, eyeball, lacrimal gland, optic nerve, temporal lobe, and brain stem.

\section{Correlation analysis}

The CT method was used to measure bilateral exophthalmos. For the bone window, the lateral zygomatic orbital process of the bilateral orbits was selected as the horizontal connection, the vertical distance $(\mathrm{cm})$ between the farthest point of the eye's ring and the horizontal line through the center of the lens was the bilateral exophthalmos (figure 1). The correlations between exophthalmos, IMRT and LOF planned for bilateral eyeball, lens, lacrimal gland, and optic nerve exposure doses were evaluated.

\section{Efficacy evaluation and observation of adverse reactions}

Fifty-eight patients were required to return to the hospital for efficacy evaluation and adverse reactions observation 6 months after radiotherapy and for annual follow-up. Efficacy evaluation indicators were vision, exophthalmos, and CAS scores, and observation indicators of adverse reactions included a comparison of dry eyes before and after treatment, vision loss, cataracts, and retinal adverse reactions.

\section{Statistical method}

SPSS 22.0 software and the independent sample's test were used to compare the differences among conformity index (CI), homogeneity index (HI), dose to $95 \%$ of the volume (D95), and PTV and the dose of endangered organs. Pearson's correlation analysis was performed for the relationship between exophthalmos and doses to the lens, eyeball, lacrimal gland, and optic nerve. $\mathrm{P}<0.05$ was considered statistically significant.

\section{RESULTS}

\section{Efficacy and adverse reactions}

Fifty-eight patients in the LOF group successfully completed all treatment plans and observation of adverse reactions. There were no special adverse reactions during the treatment. In the LOF group, 58 patients with photophobia, tearing, eye pain, and other symptoms were alleviated within 3-5 days after irradiation, and eyelid swelling was significantly reduced thereafter.

The 58 patients in the LOF group were followed up 6 months after treatment, with the result that conscious symptoms of 12 cases disappeared and 37 cases showed a significant alleviation in symptoms with an effective rate of $84 \%$. In the follow-up, conscious symptoms of six more cases significantly alleviated, and the effective rate was $95 \%$. Forty-seven patients had ocular dyskinesia before treatment, and according to the 6-month follow-up after treatment, the symptoms of 37 patients disappeared completely, the symptoms of 9 patients improved significantly, and the symptoms of 1 patient remained unchanged. Forty-nine patients had diplopia before treatment, and according to the 6-month follow-up after treatment, the symptoms of 35 patients disappeared completely, and those of 14 patients improved significantly. The exophthalmos of all patients was significantly reduced, and visual acuity was significantly improved (table 1); the CAS scores of 55 patients were significantly reduced, and those of 3 patients remained unchanged. According to the observation of adverse reactions 6 months after treatment, five patients $(8.6 \%)$ reported that they had dry eyes compared with the symptoms before treatment, and one patient (2\%) had vision loss. There were no patients with new cataracts and retinopathy. No new cases were found in the follow-up observations, with a

Table 1. Comparison before and after radiotherapy.

\begin{tabular}{|c|c|c|c|c|}
\hline Parameters $(x \pm \mathbf{s})$ & Before & After & Difference & P value \\
\hline \multicolumn{5}{|c|}{ Exophthalmos } \\
\hline Left eye & $2.37 \pm 0.32$ & $2.02 \pm 0.23$ & $0.35 \pm 0.24$ & 0.000 \\
\hline Right Eye & $2.33 \pm 0.30$ & $1.93 \pm 0.19$ & $0.40 \pm 0.20$ & 0.000 \\
\hline \multicolumn{5}{|c|}{ Vision } \\
\hline Left eye & $0.37 \pm 0.12$ & $0.54 \pm 0.09$ & $0.16 \pm 0.16$ & 0.000 \\
\hline Right Eye & $0.37 \pm 0.12$ & $0.54 \pm 0.07$ & $0.16 \pm 0.11$ & 0.000 \\
\hline CAS Score & $5.3 \pm 1.34$ & $1.3 \pm 1.03$ & $4.00 \pm 1.30$ & 0.000 \\
\hline
\end{tabular}


median follow-up time of 36 months.

\section{Comparisons of $\mathrm{CI}, \mathrm{HI}$, and doses of endangered organs}

The CI and HI of the IMRT group were better than those of the LOF group, and the differences were statistically significant. The Dmin, Dmax, D05, and D01 of PTV were higher than the LOF group, and the differences were statistically significant, while Dmean, Dmedian, and D95 were not statistically different (table 2). Doses to the lens, eyeball, temporal lobe, and brainstem were all higher than those in the LOF group, and the differences were statistically significant. Dmin, Dmean of the lacrimal gland were higher than LOF, while Dmax, D05, D01 were lower than the LOF group; the difference was statistically significant, but Dmedian had no statistical difference. Dmin of the optic nerve was lower than that of the LOF group, while Dmax, D05, and D01 were higher than those of the LOF group. The difference was statistically significant, but there was no statistical difference between Dmean and Dmedian (table 3).

\section{Correlation analysis}

Exophthalmas in the LOF group was positively correlated with the exposure dose to the lacrimal gland $(\mathrm{P}=0.000)$, was negatively correlated with the dose to the lens and eyeball $(\mathrm{P}=0.000)$, and was not correlated with the dose to the optic nerve $(\mathrm{P}=0.459,0.826)$. There was no correlation in the IMRT group $(\mathrm{P}=0.068$, $0.064,0.976,0.678,0.540,0.375,0.766,0.311$ ) (figure 2).

Table 2. Dosimetric parameters differences between PTV planned by two treatment techniques.

\begin{tabular}{|c|c|c|c|}
\hline PTV (x \pm s, cGy) & LOF & IMRT & $P$ value \\
\hline Cl & $3.59 \pm 0.80$ & $1.28 \pm 0.09$ & 0.000 \\
\hline HI & $1.13 \pm 0.09$ & $1.08 \pm 0.04$ & 0.000 \\
\hline Dmin & $1432.72 \pm 400.02$ & $2107.83 \pm 76.24$ & 0.000 \\
\hline Dmax & $2593.50 \pm 27.54$ & $2709.94 \pm 64.33$ & 0.000 \\
\hline Dmean & $2378.41 \pm 20.89$ & $2383.73 \pm 21.01$ & 0.174 \\
\hline Dmedian & $2484.07 \pm 35.93$ & $2496.24 \pm 34.09$ & 0.064 \\
\hline D95 & $2359.17 \pm 14.61$ & $2352.79 \pm 40.46$ & 0.261 \\
\hline D05 & $2553.45 \pm 42.64$ & $2619.02 \pm 44.37$ & 0.000 \\
\hline D01 & $2577.36 \pm 36.92$ & $2657.21 \pm 47.48$ & 0.000 \\
\hline
\end{tabular}

Table 3. Dosimetric parameters of OARs planned by two treatment techniques.

\begin{tabular}{|c|c|c|c|}
\hline $\begin{array}{c}\text { Parameters } \\
\text { (x } \pm \text { s, cGy) }\end{array}$ & LOF & IMRT & $P$ value \\
\hline \multicolumn{4}{|c|}{ Left lens } \\
\hline Dmin & $79.78 \pm 10.78$ & $247.95 \pm 28.01$ & 0.000 \\
\hline Dmax & $160.23 \pm 27.38$ & $418.26 \pm 54.68$ & 0.000 \\
\hline Dmean & $108.85 \pm 20.07$ & $118.85 \pm 13.48$ & 0.003 \\
\hline Dmedian & $106.04 \pm 13.44$ & $319.60 \pm 39.16$ & 0.000 \\
\hline D05 & $149.86 \pm 25.01$ & $376.39 \pm 44.09$ & 0.000 \\
\hline D01 & $157.37 \pm 25.10$ & $389.90 \pm 42.90$ & 0.000 \\
\hline \multicolumn{4}{|c|}{ Right lens } \\
\hline Dmin & $78.68 \pm 9.33$ & $248.27 \pm 23.84$ & 0.000 \\
\hline Dmax & $173.49 \pm 42.00$ & $404.74 \pm 44.41$ & 0.000 \\
\hline Dmean & $105.75 \pm 21.38$ & $119.19 \pm 13.44$ & 0.000 \\
\hline Dmedian & $106.97 \pm 14.27$ & $316.60 \pm 30.96$ & 0.000 \\
\hline D05 & $151.34 \pm 25.38$ & $375.38 \pm 38.83$ & 0.000 \\
\hline D01 & $167.07 \pm 28.04$ & $384.22 \pm 36.98$ & 0.000 \\
\hline \multicolumn{4}{|c|}{ Left eyeball } \\
\hline Dmin & $67.14 \pm 8.06$ & $190.87 \pm 30.83$ & 0.000 \\
\hline Dmax & $2475.76 \pm 51.94$ & $2505.45 \pm 95.71$ & 0.040 \\
\hline Dmean & $1107.23 \pm 187.31$ & $1278.79 \pm 128.33$ & 0.000 \\
\hline Dmedian & $919.47 \pm 170.12$ & $1328.56 \pm 104.62$ & 0.000 \\
\hline D05 & $2476.93 \pm 49.14$ & $2259.17 \pm 86.59$ & 0.000 \\
\hline D01 & $2498.46 \pm 36.29$ & $2363.41 \pm 77.85$ & 0.000 \\
\hline \multicolumn{4}{|c|}{ Right eyeball } \\
\hline Dmin & $68.67 \pm 8.30$ & $189.42 \pm 24.58$ & 0.000 \\
\hline Dmax & $2476.63 \pm 39.35$ & $2516.53 \pm 106.75$ & 0.009 \\
\hline Dmean & $1106.01 \pm 207.95$ & $1281.20 \pm 120.74$ & 0.000 \\
\hline Dmedian & $881.75 \pm 132.92$ & $1273.76 \pm 97.88$ & 0.000 \\
\hline D05 & $2483.24 \pm 46.59$ & $2242.61 \pm 94.97$ & 0.000 \\
\hline D01 & $2493.94 \pm 33.19$ & $2347.05 \pm 79.98$ & 0.000 \\
\hline \multicolumn{4}{|c|}{ Left lacrimal gland } \\
\hline Dmin & $364.01 \pm 281.27$ & $993.70 \pm 234.70$ & 0.000 \\
\hline Dmax & $2367.86 \pm 115.18$ & $2307.04 \pm 185.01$ & 0.036 \\
\hline Dmean & $1671.92 \pm 405.73$ & $1884.94 \pm 297.72$ & 0.003 \\
\hline Dmedian & $1691.81 \pm 390.03$ & $1794.72 \pm 218.27$ & 0.082 \\
\hline D05 & $2332.28 \pm 136.93$ & $2186.54 \pm 166.39$ & 0.000 \\
\hline D01 & $2412.67 \pm 68.00$ & $2239.41 \pm 173.31$ & 0.000 \\
\hline \multicolumn{4}{|c|}{ Righ lacrimal gland } \\
\hline Dmin & $278.90 \pm 142.75$ & $793.20 \pm 148.30$ & 0.000 \\
\hline Dmax & $2386.79 \pm 101.94$ & $2320.41 \pm 223.34$ & 0.042 \\
\hline Dmean & $1725.49 \pm 541.08$ & $1899.43 \pm 235.79$ & 0.036 \\
\hline Dmedian & $1757.42 \pm 275.02$ & $1699.55 \pm 185.90$ & 0.187 \\
\hline D05 & $2278.20 \pm 238.94$ & $2090.88 \pm 270.05$ & 0.000 \\
\hline D01 & $2326.62 \pm 236.63$ & $2172.38 \pm 253.72$ & 0.001 \\
\hline
\end{tabular}

Int. J. Radiat. Res., Vol. 19 No. 2, April 2021 
Continuation of Table 3. Dosimetric parameters of OARs planned by two treatment techniques.

\begin{tabular}{|c|c|c|c|}
\hline $\begin{array}{c}\text { Parameters } \\
\text { (x士s, cGy) }\end{array}$ & LOF & IMRT & $P$ value \\
\hline \multicolumn{4}{|c|}{ Left optic nerve } \\
\hline Dmin & $2351.18 \pm 53.69$ & $2259.61 \pm 66.01$ & 0.000 \\
\hline Dmax & $2523.82 \pm 31.16$ & $2589.85 \pm 37.77$ & 0.000 \\
\hline Dmean & $2450.76 \pm 147.84$ & $2464.17 \pm 44.15$ & 0.534 \\
\hline Dmedian & $2490.03 \pm 36.40$ & $2494.73 \pm 35.18$ & 0.482 \\
\hline D05 & $2524.57 \pm 36.05$ & $2559.02 \pm 32.82$ & 0.000 \\
\hline D01 & $2532.13 \pm 35.67$ & $2565.01 \pm 35.33$ & 0.000 \\
\hline \multicolumn{4}{|c|}{ Right optic nerve } \\
\hline Dmin & $2349.19 \pm 48.30$ & $2249.91 \pm 85.10$ & 0.000 \\
\hline Dmax & $2521.65 \pm 35.54$ & $2592.87 \pm 38.64$ & 0.000 \\
\hline Dmean & $2459.92 \pm 113.55$ & $2467.17 \pm 46.84$ & 0.671 \\
\hline Dmedian & $2489.04 \pm 35.48$ & $2497.67 \pm 35.26$ & 0.191 \\
\hline D05 & $2524.30 \pm 35.81$ & $2557.07 \pm 34.06$ & 0.000 \\
\hline D01 & $2530.81 \pm 34.68$ & $2567.36 \pm 37.04$ & 0.000 \\
\hline \multicolumn{4}{|c|}{ Left temporal lobe } \\
\hline Dmin & $5.18 \pm 1.06$ & $37.99 \pm 11.44$ & 0.000 \\
\hline Dmax & $2488.16 \pm 45.52$ & $2037.18 \pm 296.51$ & 0.000 \\
\hline Dmean & $164.59 \pm 30.93$ & $400.80 \pm 41.10$ & 0.000 \\
\hline Dmedian & $34.55 \pm 7.78$ & $253.08 \pm 38.80$ & 0.000 \\
\hline D05 & $1356.40 \pm 201.53$ & $1156.22 \pm 108.84$ & 0.000 \\
\hline D01 & $2333.86 \pm 60.50$ & $1527.69 \pm 202.88$ & 0.000 \\
\hline \multicolumn{4}{|c|}{ Right temporal lobe } \\
\hline Dmin & $4.74 \pm 0.71$ & $32.21 \pm 9.00$ & 0.000 \\
\hline Dmax & $2496.52 \pm 40.96$ & $2145.77 \pm 216.71$ & 0.000 \\
\hline Dmean & $184.64 \pm 33.02$ & $400.48 \pm 40.61$ & 0.000 \\
\hline Dmedian & $34.31 \pm 7.10$ & $251.27 \pm 34.10$ & 0.000 \\
\hline D05 & $1429.89 \pm 241.37$ & $1193.48 \pm 127.63$ & 0.000 \\
\hline D01 & $2390.17 \pm 53.89$ & $1593.23 \pm 192.46$ & 0.000 \\
\hline \multicolumn{4}{|c|}{ Brain stem } \\
\hline Dmin & $9.86 \pm 2.21$ & $96.69 \pm 27.49$ & 0.000 \\
\hline Dmax & $39.06 \pm 7.70$ & $1250.44 \pm 217.14$ & 0.000 \\
\hline Dmean & $18.90 \pm 4.55$ & $471.71 \pm 92.82$ & 0.000 \\
\hline Dmedian & $18.74 \pm 4.44$ & $466.32 \pm 107.99$ & 0.000 \\
\hline D05 & $29.41 \pm 6.25$ & $967.41 \pm 162.62$ & 0.000 \\
\hline D01 & $31.59 \pm 6.17$ & $1086.97 \pm 145.32$ & 0.000 \\
\hline
\end{tabular}

Int. J. Radiat. Res., Vol. 19 No. 2, April 2021
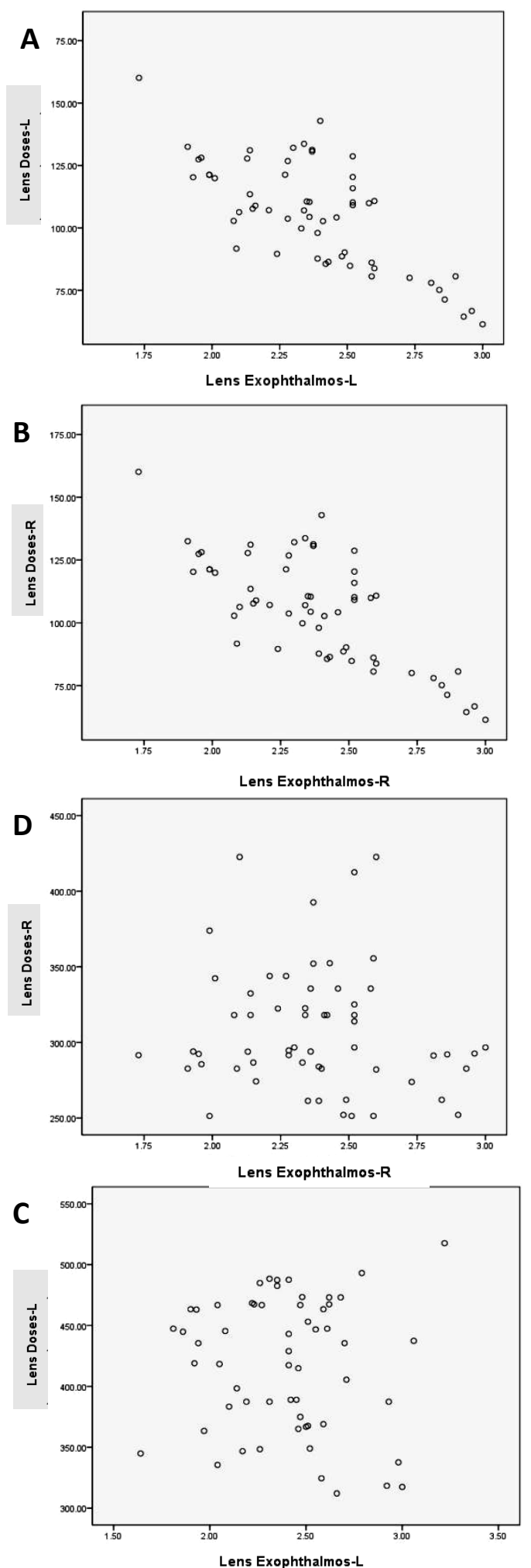

Figure 2. Correlation analysis of lens dose and exophthalmos degree by two treatment techniques. Exophthalmos degree of LOF is negatively correlated with lens dose (A, B); Exophthalmos degree of IMRT is not correlated with lens dose (C, D). 


\section{DISCUSSION}

TAO is the most common extra thyroid disease in patients with hyperthyroidism (2). The over infiltration of lymphocytes and the over production of hydrophilic glycosaminoglycans increase the amount of fat tissue and volume of extraocular muscles. Autoimmunity is considered a potential pathogenesis. For patients at the moderate or severe active stage, the first choice is high-dose glucocorticoid therapy, which can rapidly relieve symptoms, with an effective rate of only $65 \%-80 \%(15,16)$. However, it has a high incidence of adverse reactions, and patients tend to relapse after treatment is discontinued (16). Given that lymphocytes and fibroblasts infiltrated into the orbital tissues are very sensitive to radiotherapy (17), orbital radiotherapy is very effective for TAO patients (18-20) and can even be considered as an alternative therapy(21), especially for patients with hormone therapy failure or recurrence (22-25).

According to the results of several single-center retrospective studies $(18,19)$, $92 \%-97 \%$ of the patients found their condition controlled after undergoing orbital radiotherapy. This was consistent with the results of this study (95\%), and in this study, about $40 \%-60 \%$ of the patients saw a complete recovery (CR) using the NONSPECT parameter evaluation.

The reason why orbital radiotherapy cannot be widely used, at present, is the pronounced controversy regarding its potential risks and the possible side effects. The lens is one of the parts of the body most sensitive to radiation; its TD5/5 is only 1000 cGy (20). When it reaches 500 cGy, cataracts become likely to form. Over 1200 cGy, they can cause cataracts [23]. During radiotherapy, changes in the position of the eyeball can increase the dose that reaches the lens. Therefore, how to reduce the exposure dose of lens is very important in the radiotherapy of TAO.

LOF technology has been widely used in radiotherapy over the past 60 years because of its simplicity and easiness to carry out the procedure. However, in recent years, the precision radiotherapy technology represented by IMRT has significantly improved the conformability (CI) and uniformity (HI) of the target area (26). Theoretically, this can reduce the exposure dose to the lens, but this is far from confirmed. A study from Hong Kong (12) compared the dose distribution of IMRT, 3D-CRT, and LOF in the orbital radiotherapy implemented on TAO. The results showed that although IMRT had advantages in the conformability and uniformity of target area, it was worse than the LOF technology in terms of organ protection, planning time, execution efficiency, and number of hops (MU). The results of this study also confirm this. In addition, LOF was significantly superior to IMRT in reducing the exposure dose to the lens and other organs at risk, which be related to the exophthalmos in patients with TAO. LOF technology can use this feature to draw the protruding lens far away from the target area behind the eyeball, and then reduce the exposure dose. The radiation field of the technology has two centers of irradiation of equal size, which can avoid any need to increase the dose that can occur when the lens changes position with the movement of the eyeball in the process of radiotherapy. Furthermore, the front field lead block of the LOF technology can effectively reduce the scattering caused by the multi-leaf grating (MLC). Subsequent correlation analysis showed that the dose in the LOF group was negatively correlated with the degree of exophthalmos $(P=0.000)$, while this was not so in the IMRT group. This study suggests that LOF may bring more benefits to TAO patients with large exophthalmos than IMRT. Also, it is superior to IMRT in terms of reducing the exposure dose of eyeball, optic nerve, lacrimal gland, temporal lobe, brain stem, and other organs at risk, although the tolerance of these organs to radiation is much higher than the total dose of TAO (2000-2400 cGy). At present, no adverse reactions have been reported in clinical settings, but long-term toxicity requires follow-up observation. It should also be noted that both the execution efficiency and MU of LOF are significantly better than those of IMRT, which shortens the treatment time, reducing the possibility that the patient's head will become 
involuntarily displaced, further improving the treatment accuracy and comfort. In addition to the above advantages, because the cost of radiotherapy changes depending on the number of radiation fields, the LOF of 2 fields is also more economical than that of IMRT with 7 fields.

According to a previous clinical report that the most common adverse reaction of retrobulbar radiotherapy is dry eyes (12\%) (18), which is slightly higher than our results $(8.6 \%)$. However, compared with the improvement of eye symptoms, dry eyes were acceptable, and symptoms of dry eyes of all patients had improved after treatment with artificial tears. Other complications such as cataracts and retinopathy were very low in reports from multiple centers. In the follow-up of this study, the eye symptoms of one patient improved but with lower vision than before, considering other confounding factors that may be associated with older age. The second primary tumor caused by radiation is the most serious and unacceptable complication of radiotherapy for benign diseases. There is no real case report on this at present. The theoretical risk of long-term survival predicted by a risk model is $0.7 \%$ (27). Additional attention is still needed, which requires long-term follow-up observation.

In summary, the two-field paired irradiation can make full use of the characteristics of TAO to reduce the dose of radiation that reaches the lens and other at-risk organs. This can also shorten the treatment time, thereby reducing the physiological, psychological, and economic pressure on patients. No serious adverse reactions occurred during the follow-up, which can be used as a basis for the development of disease risk-benefit models, but more clinical data are still needed to support the study due to the short follow-up time involved here.

\section{ACKNOWLEDGEMENT}

This work was supported by grants from Special clinical characteristic research project of health development in Tongzhou District, Beijing (No. KJ2019CX 012-33). The funding bodies had no role in the design of the study or collection, analysis, and interpretation of the data; or writing the manuscript. We thank LetPub (www.letpub.com) for its linguistic assistance during the preparation of this manuscript.

\section{Conflicts of interest: Declared none.}

\section{REFERENCES}

1. Marcocci C (2018) Graves'Ophthalmopathy. West J Med, 158(6): 591.

2. Bartalena L, Baldeschi L, Dickinson A, et al. (2008) Consensus statement of the European Group on Graves' orbitopathy (EUGOGO) on management of GO. Eur J Endocrinol, 158(3): 273-285

3. Bartalena L, Baldeschi L, Boboridis K, et al. (2016) The 2016 European Thyroid Association/European Group on Graves' Orbitopathy Guidelines for the Management of Graves' Orbitopathy. Eur Thyroid J, 5(1): 9-26.

4. Bartalena L, Krassas GE, Wiersinga, W, et al. (2012) Efficacy and safety of three different cumulative doses of intravenous methylprednisolone for moderate to severe and active Graves' orbitopathy. J Clin Endocrinol Metab, 97 (12): 4454-63.

5. Wang L and Ma JM (2017) Progression of the pathogenesis of thyroid associated ophthalmopathy. Chinese J Ophthalmol, 53(6): 474-80.

6. Traisk F, Tallstedt L, Abraham-Nordling M, et al. (2009) Thyroid-associated ophthalmopathy after treatment for Graves' hyperthyroidism with antithyroid drugs or iodine131. J Clin Endocrinol Metab, 94(10): 3700-7.

7. Matthiesen C, Thompson JS, Thompson D, et al. (2012) The efficacy of radiation therapy in the treatment of Graves' orbitopathy. Int J Radiat Oncol Biol Physic, 82 (1): 117-123.

8. Prabhu RS, Liebman L, Wojno T, et al. (2012) Clinical outcomes of radiotherapy as initial local therapy for Graves' ophthalmopathy and predictors of the need for postradiotherapy decompressive surgery. Radiat Oncol, 7(1): 95-95.

9. Marquez SD, Lum BL, McDougall IR, et al. (2001) Longterm results of irradiation for patients with progressive Graves' ophthalmopathy. Int J Radiat Oncol Biol Phys, 51 (3): 766-74.

10. Schaefer U, Hesselmann S, Micke O, et al. (2002) A longterm follow-up study after retro-orbital irradiation for Graves' ophthalmopathy. Int J Radiat Oncol Biol Phys, 52 (1): 192-7.

11. Li Yim JF, Sandinha T, Kerr JM, et al. (2011) Low dose orbital radiotherapy for thyroid eye disease. Orbit, 30(6): 269-74.

12. Lee VH, Ng SC, Choi CW, et al. (2012) Comparative analysis of dosimetric parameters of three different radiation techniques for patients with Graves' ophthalmopathy treated with retro-orbital irradiation. Radiat Oncol, 7: 199. 


\section{Nan et al. / Dosimetric comparison of lateral opposing fields in TAO irradiation}

13. San-Miguel I, Carmona R, Luque, L, et al. (2016) Volumetric Modulated Arc Therapy (VMAT) make a difference in retro-orbital irradiation treatment of patients with bilateral Graves' ophthalmopathy. Comparative analysis of dosimetric parameters from different radiation techniques. Rep Pract Oncol Radiother, 21(5): 435-40.

14. Li YJ, Luo Y, Xie XQ, et al. (2017) The efficacy of intensity modulated radiation therapy in treating thyroidassociated ophthalmopathy and predictive factors for treatment response. Sci Rep, 7(1): 17533.

15. Zang S, Ponto KA, Kahaly GJ (2011) Clinical review: Intravenous glucocorticoids for Graves' orbitopathy: efficacy and morbidity. J Clin Endocrinol Metab, 96(2): 320-32.

16. San Miguel I, Arenas M, Carmona R, et al. (2018) Review of the treatment of Graves' ophthalmopathy: The role of the new radiation techniques. Saudi J Ophthalmol, 32(2): 13945

17. Wiersinga WPM (1995) Therapeutic Controversies. Retrobulbar Radiation in Graves' Ophthalmopathy. Clin Endocrinol Metab, 80(2): 345-7.

18. Matthiesen C, Thompson JS, Thompson D, et al. (2012) The efficacy of radiation therapy in the treatment of Graves' orbitopathy. Int I Radiat Oncol Biol Phys, 82(1): 117-23.

19. Prabhu RS, Liebman L, Wojno T, et al. (2012) Clinical outcomes of radiotherapy as initial local therapy for Graves' ophthalmopathy and predictors of the need for postradiotherapy decompressive surgery. Radiation Oncology, 7: 95.
20. Emami B, Lyman J, BrownA, et al. (1991) Tolerance of normal tissue to therapeutic irradiation. Int J Radiat Oncol Biol Phys, 21(1): 109-22.

21. Bradley EA, Gower EW, Bradley DJ, et al. (2008) Orbital radiation for graves ophthalmopathy: a report by the American Academy of Ophthalmology. Ophthalmology, 115(2): 398-409.

22. Prummel MF, Terwee CB, Gerding MN, et al. (2004) A randomized controlled trial of orbital radiotherapy versus sham irradiation in patients with mild Graves' ophthalmopathy. J Clin Endocrinol Metab, 89(1): 15-20.

23. Ferrufino-Ponce ZK and Henderson BA (2006) Radiotherapy and cataract formation. Semin Ophthalmol, 21(3): 17180.

24. Kazim M and Garrity JA (2012) Orbital radiation therapy for thyroid eye disease. J Neuro-Ophthalmol, 32(2): 172-6.

25. Mourits MP, van Kempen-Harteveld ML, Garcia MBG, et al. (2000) Radiotherapy for Graves' orbitopathy: randomised placebo-controlled study. Lancet, 355(9214): 1505-9.

26. Jian N, X-FMU, Jie BU, et al. (2011) Dosimetry study of different irradiation technologies for patients with recurrence of cervical cancer. Medic J Chinese People's Liberation Army, 36(7): 742-4.

27. Broerse JJ, Snijders-Keilholz A, Jansen JT, et al. (1999) Assessment of a carcinogenic risk for treatment of Graves' ophthalmopathy in dependence on age and irradiation geometry. Radiother Oncol, 53(3): 205-8. 